\title{
Algorithmic Analysis of Nonlinear Hybrid Systems
}

\author{
Thomas A. Henzinger and Pei-Hsin Ho \\ Computer Science Department, Cornell University, Ithaca, NY 14853 \\ (tah|ho)@cs.cornell.edu
}

\begin{abstract}
Hybrid systems model discrete programs that are embedded in continuous environments. Model-checking tools are available for the analysis of linear hybrid systems, whose continuous variables are bounded by piecewiselinear trajectories. Most embedded programs, however, operate in nonlinear environments. We present, analyze, and apply two algorithms for translating nonlinear hybrid systems into linear hybrid systems.

The clock translation replaces nonlinear variables by clock variables; the rate translation approximates nonlinear variables by piecewise-linear envelopes. Both translations are sound for reachability; that is, if we establish a safety property of the translated linear system, we may conclude that the original nonlinear system satisfies the property. The clock translation is also complete for reachability; that is, the original system and the translated system satisfy the same safety properties. The two translations apply to incomparable classes of nonlinear hybrid systems. From the clock translation we obtain a new decidability result for hybrid systems.

With the help of HYTECH, a symbolic model checker for linear hybrid systems, we automatically verify a nonlinear railroad gate control program using the clock translation, and a nonlinear temperature control program using the rate translation.
\end{abstract}

\section{Introduction}

Hybrid systems are digital real-time systems that are embedded in analog environments. Due to the rapid development of digital-processor technology, hybrid systems directly control much of what we depend on in our daily lives. Many hybrid systems, ranging from automobiles to aircraft, operate in safety-critical situations and therefore call for rigorous analysis techniques. Consequently, the formal specification and verification of hybrid systems has become an active area of research [7]. The symbolic model-checking approach, in particular, has been successfully extended from discrete systems [5] to real-time systems [9] and linear hybrid systems [1, 4]. While the symbolic model-checking method developed in these papers is limited to piecewise-linear dynamics, many typical real-time parameters (e.g., temperature) behave in a nonlinear fashion. The present paper extends the model-checking approach to the analysis of certain nonlinear systems. ${ }^{2}$

* This research was supported in part by the NSF grant CCR-9200794, by the AFOSR contract F49620-93-1-0056, and by the DARPA grant NAG2-892.

2 Control theory, of course, has a long tradition of analyzing what we call nonlinear hybrid systems (in control theory, the term linearity usually refers to differential equations, not trajectories). There, however, the number of discrete modes of a controller is typically quite small. Model checking, on the other hand, allows the analysis of controllers that are defined by arbitrary finite-state programs. While the control theorists start with complex environments-differential equations-and steadily in- 
We model hybrid systems as hybrid automata [2,10] (Section 2). A hybrid automaton operates with a finite control over a data space of both discrete and continuous variables. The discrete variables are updated by automaton transitions; the continuous variables evolve according to differential equations. In [4], we introduced the symbolic model checker HYTECH (the Cornell Hybrid Technology Tool) for analyzing linear hybrid automata. Here, we turn to the analysis of nonlinear hybrid automata, which alone provide an accurate model for most real-time environments (for example, the temperature of a furnace decreases along an exponential curve with negative exponent). ${ }^{3}$

A hybrid automaton defines an infinite-state transition system, and the reachability analysis of a hybrid automaton requires the computation of weakest preconditions in the underlying transition system. As we know how to compute weakest preconditions accurately only for linear hybrid automata, we propose a two-step methodology for verifying a nonlinear hybrid automaton $A$. In Step 1 , we translate $A$ into a linear hybrid automaton $B$. In Step 2, we apply the HYTECH tool to the translated automaton $B$. The translation is $\mathcal{P}$-sound, for a class $\mathcal{P}$ of properties, if all $\mathcal{P}$-properties of $B$ are inherited by $A ; \mathcal{P}$-complete, if all $\mathcal{P}$-properties of $A$ are inherited by $B$. Incomplete translations may lead to false negatives: $B$ may not satisfy the $\mathcal{P}$-property $\varphi$ although $A$ does. We therefore accompany incomplete translations with error analyses: given a metric $d$ on hybrid automata, what is the automaton $A^{\prime} d$-closest to $A$ such that if $A^{\prime}$ satisfies $\varphi$, then so does $B$ ? If the $d$-difference between $A$ and $A^{\prime}$ can be made arbitrarily small, then the translation is called asymptotically $\mathcal{P}$-complete under the metric $d$.

We present two translations, which transform two incomparable classes of nonlinear hybrid automata into linear hybrid automata. The clock translation (Section 3) replaces nonlinear variables by clocks (i.e., linear variables with slope 1). The clock translation is applicable to the nonlinear variable $x$ if the value of $x$ is always uniquely determined by the latest assignment to $x$ and the time that has expired since that assignment. If the clock translation of the automaton $A$ yields the automaton $B$, then the underlying transition systems are timed bisimilar. It follows that the clock translation is both sound and complete for all branching-time properties. If all variables of a nonlinear hybrid automaton can be replaced by clocks, then the resulting linear hybrid automaton is a timed automaton [3]. As a corollary, we obtain a new decidable class of hybrid automata. We verify a nonlinear railroad gate controller using the clock translation and the HYTECH model checker.

The rate translation (Section 4) approximates nonlinear variables by piecewiselinear variables. The rate translation is applicable to the nonlinear variable $x$ if the value of $x$ is bounded. If the rate translation of the automaton $A$ yields the automaton $B$, then the transition system of $B$ simulates the transition system of $A$ (but not vice versa). It follows that the rate translation, while sound for all linear-time properties, is not complete for safety properties. We show that the rate translation is asymptotically complete for safety properties. We verify a nonlinear temperature controller using the rate translation and the HYTECH model checker. Technically, both the clock translation and the rate translation can be viewed as abstract interpretations of nonlinear hybrid automata [6].

crease the complexity of the controllers that can be analyzed, we computer scientists start with complex controllers-programs!-and steadily increase the complexity of the environments.

${ }^{3}$ We insist on representing nonlinear behavior accurately in our underlying model, because we feel that linearization or digitization ought to occur after the modeling phase, so that the errors that are introduced by these processes can be analyzed and bounded. Such an analysis, indeed, is performed in the present paper for both linearizations we propose. 


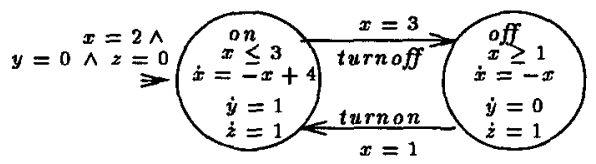

Fig. 1. Thermostat automaton

\section{Hybrid Automata}

Informally, a hybrid automaton consists of a finite vector $\mathbf{x}$ of real-valued variables and a labeled multigraph $(V, E)$. The edges $E$ represent discrete system actions and are labeled with nondeterministic guarded assignments to $\mathbf{x}$. The vertices $V$ represent continuous environment activities and are labeled with constraints on the derivatives of $\mathrm{x}$. The state of the automaton changes either through instantaneous system actions or, while time elapses, through continuous activities.

For example, the hybrid automaton of Figure 1 models a thermostat that controls the temperature of a manufacturing plant. The nonlinear variable $x$ represents the plant temperature. In control location on, a heater is turned on; in control location off, the heater is turned off. The variable $x$ follow the differential equations $\dot{x}=-x+4$ in location on and $\dot{x}=-x$ in location off. Initially, the temperature is 2 and the heater is turned on. If a thermometer detects that the plant temperature reaches 3 , the heater is turned off. If the thermometer detects that the temperature falls to 1 , the heater is turned on. The two variables $y$ and $z$ are auxiliary variables: $y$, a stop watch, records the accumulated time that the heater is turned on, and $z$, a clock, records the total elapsed time.

Syntax Let $\mathbf{y}$ be a vector of real-valued variables. A linear term over $\mathbf{y}$ is a linear combination of variables from $\mathrm{y}$ with real coefficients. ${ }^{4} \mathrm{~A}$ linear inequality over $\mathrm{y}$ is a nonstrict inequality between linear terms over $y$. A hybrid automaton $A$ consists of the following components [2]:

Data variables $\mathrm{A}$ finite vector $\mathbf{x}=\left(x_{1}, \ldots, x_{n}\right)$ of real-valued data variables. The thermostat automaton in Figure 1 has the vector $(x, y, z)$ of data variables. A data state is a point in $n$-dimensional space $\mathbb{R}^{n}$. A convex data region is a convex polyhedron in $\mathrm{IR}^{n}$; a data region is a finite union of convex data regions. A convex data predicate is a conjunction of linear inequalities (e.g., $x_{3} \leq 2 x_{2}-1 \wedge 6 \leq x_{3}$ ). A data predicate is a disjunction of convex data predicates. Each (convex) data predicate $\phi$ defines, then, a (convex) data region $[\phi] \subseteq \mathbb{R}^{n}$ such that $\mathbf{s} \in[\phi]$ iff $\phi[\mathbf{x}:=\mathbf{s}]$ is true.

An action predicate is a conjunction of linear inequalities over $x \cup x^{\prime}$, where $x^{\prime}=$ $\left(x_{1}^{\prime}, \ldots, x_{n}^{\prime}\right)$ is the vector of primed data variables. Each action predicate $\psi$ defines a function from data states $s \in \mathbb{R}^{n}$ to convex data regions $[\psi](\mathbf{s}) \subseteq \mathbb{R}^{n}: \mathbf{s}^{\prime} \in$ $\llbracket \psi \rrbracket(\mathbf{s})$ iff $\psi\left[\mathbf{x}, \mathbf{x}^{\prime}:=\mathbf{s}, \mathbf{s}^{\prime}\right]$ is true.

Control locations A finite set $V$ of vertices called locations. The thermostat automaton has the locations on and off. A state $(v, \mathrm{~s})$ of the hybrid automaton $A$ consists of a location $v \in V$ and a data state $s \in \mathbb{R}^{n}$. A region $\bigcup_{v \in V}\left\{\left(v, S_{v}\right)\right\}$ is a collection of data regions $S_{v} \subseteq \mathbb{R}^{n}$, one for each location $v \in V$. A state predicate is a collection $\bigcup_{v \in V}\left\{\left(v, \phi_{v}\right)\right\}$ of data predicates $\phi_{v}$, one for each location $v \in V$. When writing state predicates, we omit disjuncts of the form $(v$, false). Each state predicate $\varphi=\bigcup_{v \in V}\left\{\left(v, \phi_{v}\right)\right\}$ defines, then, the region $\llbracket \varphi \rrbracket=\bigcup_{v \in V}\left\{\left(v, \llbracket \phi_{v} \rrbracket\right)\right\}$.

${ }^{4}$ We shall specify coefficients by symbolic expressions like $\ln 3$. 
Initial conditions $\mathrm{A}$ labeling function init that assigns to each location $v \in V$ a convex data predicate init $(v)$, the initial condition of $v$. The control of $A$ may start in the location $v$ only when the initial condition init $(v)$ is true. In the graphical representation of hybrid automata, we usually suppress initial condition of the form false. In the thermostat automaton, the initial conditions of the locations on and off are $x=2 \wedge y=0 \wedge z=0$ and false, respectively. We write $I_{A}$ for the initial region $\bigcup_{v \in V}\{(v,[\operatorname{init}(v)])\}$ of $A$.

Location invariants $A$ labeling function inv that assigns to each location $v \in V$ a convex data predicate $i n v(v)$, the invariant of $v$. The control of $A$ may reside in the location $v$ only while the invariant $i n v(v)$ is true. In the graphical representation, we usually suppress invariants of the form true. In the thermostat automaton, the invariants of the locations on and off are $x \leq 3$ and $x \geq 1$, respectively. We write $\Sigma_{A}$ for the admissible region $\bigcup_{v \in V}\{(v,[i n v(v)])\}$ of $A$.

Continuous activities A labeling function dif that assigns to each location $v \in V$ and each data variable $x_{i}$ an activity dif $\left(v, x_{i}\right)$, which is either (1) a linear term $\operatorname{dif}\left(v, x_{i}\right)=f(\mathrm{x})$ over $\mathrm{x}$; or (2) a rate interval dif $\left(v, x_{i}\right)=[a, b]$, which is a bounded or unbounded, closed interval of the real line with $\pm \infty$ (that is, $a, b \in \mathbb{R} \cup\{-\infty, \infty\}$ and $a \leq b)$. In the first case, if the control of $A$ resides in the location $v$, the value of $x_{i}$ evolves according to the first-order differential equation $\dot{x}_{i}=f(\mathbf{x})$. In the second case, the interval endpoints $a$ and $b$ give $a$ lower bound and an upper bound on the derivative of $x_{i}$; that is, $a \leq \dot{x}_{i} \leq b$.

The data variable $x$ is linear if for all locations $v \in V$, dif $(v, x)$ is a rate interval; otherwise, $x$ is nonlinear. The hybrid automaton $A$ is linear if all data variables in $\mathbf{x}$ are linear [4]; otherwise, $A$ is nonlinear. In the thermostat automaton, the data variables $y$ and $z$ are linear. The variable $x$ is a nonlinear variable with $\operatorname{dif}($ on,$x)=-x+4$ and $\operatorname{dif}($ off,$x)=-x$.

Edges A finite multiset $E$ of edges. Each edge $\left(v, v^{\prime}\right)$ identifies a source location $v \in V$ and a target location $v^{\prime} \in V$. For each location $v \in V$, there is a stutter edge $e_{v}=(v, v)$. In the graphical representation, we usually omit stutter edges. The thermostat automaton has four edges: (on, off), (off, on $),($ on, on $)$, and (off, off).

Discrete actions $A$ labeling function act that assigns to each edge $e \in E$ a convex action predicate $a c t\left(v, v^{\prime}\right)$, the action of $e$. If the control of $A$ proceeds from the location $v$ to the location $v^{\prime}$ via the edge $e=\left(v, v^{\prime}\right)$, then the values of all data variables change from $s$ nondeterministically to any point within the data region $[\operatorname{act}(e)](\mathrm{s})$. For example, an edge with the action label $x_{1} \leq 3 \wedge 3 \leq x_{1}^{\prime} \leq$ $5 \wedge x_{2}^{\prime}=x_{2} \wedge x_{3}^{\prime}=x_{1}+1$ can be traversed only when the value of $x_{1}$ is at most 3 . By traversing the edge, the value of $x_{1}$ changes to any real number in the interval $[3,5]$, the value of $x_{2}$ remains unchanged, and the new value of $x_{3}$ is 1 plus the old value of $x_{1}$. The stutter edges are labeled with the action true. In the graphical representation of actions, if the primed variable $x^{\prime}$ occurs only in the conjunct $x^{\prime}=x$, then we usually omit that conjunct. In the thermostat automaton, $\operatorname{act}($ on, off $)$ is $x=3 \wedge x^{\prime}=x \wedge y^{\prime}=y \wedge z^{\prime}=z$.

Edge labels A finite set $L$ of edge labels and a labeling function label that assigns to each edge $e \in E$ a label from $L$. For all stutter edges $e_{v}, \operatorname{label}\left(e_{v}\right)=v$. The edge labels are used to define the parallel composition of hybrid automata. In the thermostat automaton, label (on, off) is turnoff.

Accepting conditions A labeling function final that assigns to each location $v \in V$ a data predicate final $(v)$, the accepting condition of $v$. We use the accepting conditions to check safety properties of hybrid automata. In the graphical representation of hybrid automata, we suppress accepting conditions of the form true. We write $F_{A}$ for the accepting region region $\bigcup_{v \in V}\{(v,[$ final $(v)])\}$ of $A$. 
The data or action predicate $\phi$ is rational if all constants that occur in $\phi$ are rational. The hybrid automaton $A$ is rational if all data and action predicates that specify the initial and accepting conditions, invariants, and actions of $A$ are rational. A state predicate is rational if it contains only rational data predicates.

A special case of a linear hybrid automaton is a timed automaton [3]. The data variable $x$ of the hybrid automaton $A$ is a clock if $\operatorname{dif}(v, x)=[1,1]$ for all locations $v$ of $A$; that is, each clock always increases with the rate at which time advances. An atomic data predicate is simple if it has the form $x \leq c$ or $x \geq c$, for some $c \in \mathbb{R}$; an atomic action predicate is simple if it is a simple atomic data predicate or has the form $x^{\prime}=c$ or $x^{\prime}=x$. The data variable $x$ of $A$ is simple if in all initial and accepting conditions, invariants, and actions of $A, x$ and $x^{\prime}$ occur only in atomic data and action predicates that are simple. The hybrid automaton $A$ is a timed automaton if all data variables of $A$ are simple clocks.

Semantics At any time instant, the state of a hybrid automaton $A$ specifies a location and values for all data variables. The state can change in two ways: (1) by an instantaneous move through an edge that changes both the location and the values of data variables according to the corresponding action; or (2) by a time delay that changes only the values of data variables in a continuous manner according to the activities of the current control location. A data trajectory $(\delta, \rho)$ in location $v$ of the hybrid automaton $A$ consists of a nonnegative duration $\delta \in \mathbb{R} \geq 0$ and a differentiable function $\rho:[0, \delta] \rightarrow \mathbb{R}^{n}$ such that

1. for all reals $t \in[0, \delta], \rho(t) \in[$ inv $(v)]$;

2. for each data variable $x_{i}$ with $\operatorname{dif}\left(v, x_{i}\right)=f(\mathbf{x})$, for all reals $t \in(0, \delta), d \rho\left(x_{i}\right)(t) / d t=$ $f(x)$ (where $\rho\left(x_{i}\right)(t)$ denotes the $i$ th component of the data state $\rho(t)$ ); and

3. for each data variable $x_{i}$ with $\operatorname{dif}\left(v, x_{i}\right)=[a, b]$, for all reals $t \in(0, \delta), d \rho\left(x_{i}\right)(t) / d t \in$ $[a, b]$.

We define the the following two transition relations on the admissible states of the hybrid automaton $A$ :

Edge step For all states $\sigma_{1}=\left(v, \mathbf{s}_{1}\right)$ and $\sigma_{2}=\left(v^{\prime}, \mathbf{s}_{2}\right)$ of $A$, and all edge labels $\ell$, $\sigma_{1} \stackrel{\ell}{\rightarrow} \sigma_{2}$ if $\sigma_{1}, \sigma_{2} \in \Sigma_{A}$, and there exists an edge $e$ from $v$ to $v^{\prime}$ such that label $(e)=\ell$ and $s_{2} \in[\operatorname{act}(e)]\left(s_{1}\right)$.

Time step For all states $\sigma_{1}=\left(v, \mathbf{s}_{1}\right)$ and $\sigma_{2}=\left(v, \mathbf{s}_{2}\right)$ of $A$, and all durations $\delta \in \mathbb{R}_{\geq 0}$, $\sigma_{1} \stackrel{\delta}{\rightarrow} \sigma_{2}$ if there exists a data trajectory $(\delta, \rho)$ in location $v$ such that $\rho(0)=\mathbf{s}_{1}$ and $\rho(\delta)=\mathbf{s}_{2}$.

The hybrid automaton $A$ defines the labeled transition system $[A]=\left\langle\Sigma_{A}, I_{A}, \mathcal{L}, \rightarrow_{A}\right.$ ,$\left.F_{A}\right)$ that consists of (1) the infinite state space $\Sigma_{A},(2)$ the set $I_{A}$ of initial states, (3) the alphabet $\mathcal{L}=L \cup \mathbb{R}_{\geq 0}$, (4) the transition relation $\rightarrow_{A}=\bigcup\{\stackrel{\delta}{\rightarrow} \mid \delta \geq 0\} \cup$ $\bigcup\{\stackrel{\ell}{\rightarrow} \mid \ell \in L\}$, and $(5)$ the set $F_{A}$ of accepting states. A trajectory $\tau$ of $A$ is a finite path $\sigma_{0} \stackrel{m_{0}}{\rightarrow} \sigma_{1} \stackrel{m_{1}}{\rightarrow} \ldots \stackrel{m_{k-1}}{\rightarrow} \sigma_{k}$ in $[A]$ such that $\sigma_{0} \in I_{A}$ and for all $i \in\{0, \ldots, k-1\}$, $\left(\sigma_{i} \stackrel{m_{i}}{\rightarrow} \sigma_{i+1}\right) \in \rightarrow_{A}$. The trajectory $\tau$ is accepting if the final state of $\tau$ is accepting; that is, $\sigma_{k} \in F_{A}$. The reachable region $R(A) \subseteq \Sigma_{A}$ of the hybrid automaton $A$ is the set of all final states on trajectories of $A$.

Parallel composition A hybrid system typically consists of many components that operate concurrently and communicate with each other. We describe each component as a hybrid automaton. The edge labels can be used to synchronize various system components. The hybrid automaton that models the entire system is then constructed from the component automata using a product operation that is defined in the standard way [4]. 


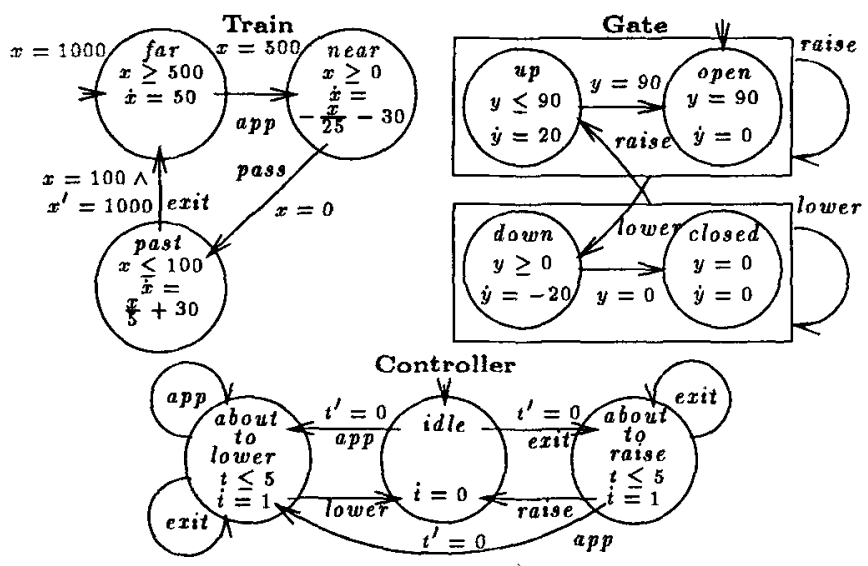

Fig. 2. A railroad gate controller

Example: Nonlinear railroad gate controller We present a nonlinear variation of a familiar example [4]. The three hybrid automata of Figure 2 model three processes-a train, a gate, and a controller. The nonlinear variable $x$ represents the distance of the train from the gate. Initially, the train is far from the gate and moves at the speed 50 meters per second. When the train approaches the gate, a sensor placed at a distance of 500 meters from the crossing detects the train and sends the signal app to the controller. The train starts to slow down and the controller waits 5 seconds before sending the command lower to the gate; the delay of the controller is modeled by the clock $t$. Consequently, the gate is lowered from 90 radius degrees to 0 degrees at the constant rate of 20 degrees per second; the position of the gate in degrees is represented by variable $y$. After passing the gate, the train begins to accelerate. A second sensor placed at 100 meters past the crossing detects the leaving train and signals exit to the controller, which, after another delay of 5 seconds, sends the command raise to the gate. The distance between consecutive trains is (at least) 1,000 meters. Notice that the synchronization signals like app, exit and raise are modeled by edge labels.

The emptiness problem The emptiness problem for hybrid automata asks, given a hybrid automaton $A$, if $A$ has an accepting trajectory. If the accepting region $F_{A}$ represents the set of "unsafe" states specified by a safety property, then the safety property can be verified by checking the emptiness of $A$, namely, the safety property is satisfied by $A$ iff $A$ has no accepting trajectory. The emptiness problem is decidable for rational timed automata [3] and certain rational simple linear hybrid automata [8], and semidecidable for rational linear hybrid automata [2].

For the thermostat automaton of Figure 1, we will verify the safety property whose "unsafe" region is characterized by the state predicate $\bigcup_{v \in\{o n, o f f\}}\{(v, 6 \leq z \leq 2 y-1)\}$; that is, after 6 time units the heater has always been on at most half of the time plus 1 time unit. For the railroad gate controller of Figure 2, we will verify the safety property whose unsafe region is characterized by the state predicate $\bigcup_{v \not \neq c l o s e d}\{(v, x \leq 100)\}$; that is, whenever the train is within 100 meters from the gate, the gate is closed (we write $v \neq$ closed if the gate component of location $v$ is closed). 


\section{Clock Translation}

The clock translation of a hybrid automaton replaces each nonlinear data variable $x$ by a clock $t_{x}$ that is restarted whenever the value of $x$ is changed by a discrete action. The clock translation is applicable if at every point of a trajectory, the value of $x$ is uniquely determined by the value of $t_{x}$.

Solvable automata Let $A$ be a hybrid automaton. The simple nonlinear data variable $x$ of $A$ is (rationally) determined in the location $v$ of $A$ if dif $(v, x)=f(x)$ and for all (rational) initial values $x_{0} \in \mathbb{R}$, the initial-value problem " $\dot{x}(t)=f(x) ; x(0)=x_{0}$ " has an algebraic solution $x_{v, x_{0}}(t)$ such that for each constant $c$ that appears in an atomic data predicate $x \sim c$, for $\sim \in\{\leq, \geq\}$, or an atomic action predicate $x^{\prime}=c$ of $A$, the function $x_{v, x_{0}}(t)-c$ has a finite number of (rational) roots. For example, suppose that the function $x_{v, x_{0}}(t)-c_{0}$ has the two roots $r_{0}$ and $r_{1}$, and all trajectories that enter the location $v$ have the initial value $x_{0}$ for $x$. Then the value of the variable $x$ is $x_{v, x_{0}}\left(t_{x}\right)$ for all reachable states in $v$. Thus an exit edge of location $v$ guarded with $x=c_{0}$ can be replaced by two exit edges guarded with $t_{x}=r_{0}$ and $t_{x}=r_{1}$, respectively.

The location $v$ of $A$ is definite for the data variable $x$ if the initial condition init(v) implies $x=c$, for some initial value $c \in \mathbb{R}$. The edge $e$ of $A$ is definite for $x$ if the action act $(e)$ implies $x^{\prime}=c$, for some arrival value $c \in \mathbb{R}$. The simple nonlinear data variable $x$ of $A$ is solvable if the following three conditions hold:

1. For all locations $v$ of $A, x$ is determined in $v$.

2. All locations of $A$ are definite for $x$.

3. For all edges $e=\left(v, v^{\prime}\right)$ of $A$, if $\operatorname{dif}(v, x) \neq \operatorname{dif}\left(v^{\prime}, x\right)$, then $e$ is definite for $x$;

For example, the nonlinear variable $x$ of the thermostat automaton of Figure 1 is solvable, and so is the the nonlinear variable $x$ of the train automaton of Figure 2 . The hybrid automaton $A$ is solvable if all nonlinear data variables of $A$ are simple and solvable. The automaton $A$ is rationally solvable if $A$ is (1) rational, (2) solvable, and (3) all data variables of $A$ are rationally determined in all locations of $A$. All (rational) timed automata are (rationally) solvable and the class of (rationally) solvable hybrid automata is closed under parallel composition. For each solvable data variable $x$, we collect the initial values of $x$ for all locations and the arrival values of $x$ for all definite edges in the finite set $C$ rit $\operatorname{Val}(x) \subseteq \mathbb{R}$ of critical values for $x$.

The clock translation algorithm Given a solvable nonlinear hybrid automaton $A$, we construct a linear hybrid automaton $A^{c}$ - the clock translation of $A$-by replacing each nonlinear data variable $x$ with a new clock $t_{x}$. For each nonlinear data variable $x$, the construction proceeds in two steps:

1. Let $\operatorname{Crit\operatorname {Val}}(x)=\left\{c_{1}, \ldots, c_{n}\right\}$ with $c_{1}<\cdots<c_{n}$. Each location $v$ of $A$ is split into a collection $v_{c_{1}}, \ldots, v_{c_{n}}$ of locations, one for each critical value $c_{i}$ of $x$. We then add the clock $t_{x}$ such that the value of $x$ in location $v_{c_{i}}$ is $x\left(t_{x}\right)$, where $x(t)$ is the solution of the initial-value problem " $\dot{x}(t)=\operatorname{dif}(v, x) ; x(0)=c_{i}$ ".

2. All initial and accepting conditions, invariants, and actions are translated from conditions on $x$ to conditions on $t_{x}$.

We now provide more details.

Step 1. Splitting locations and edges After the application of Step 1, each new location $v_{c_{i}}$ has the same activities as $v$ and, in addition, the new activity $\operatorname{dif}\left(v_{c_{i}}, t_{x}\right)=1$ for the clock $t_{x}$. The new location $v_{c_{i}}$ has the the initial condition $\operatorname{init}(v) \wedge t_{x}=0$, the accepting condition $f$ inal $(v)$, and the invariant inv $(v)$. For each indefinite edge $e=\left(v, v^{\prime}\right)$, we introduce all edges of the form $\left(v_{c_{i}}, v_{c_{i}}^{\prime}\right)$ with the action act $(e)$ and the 


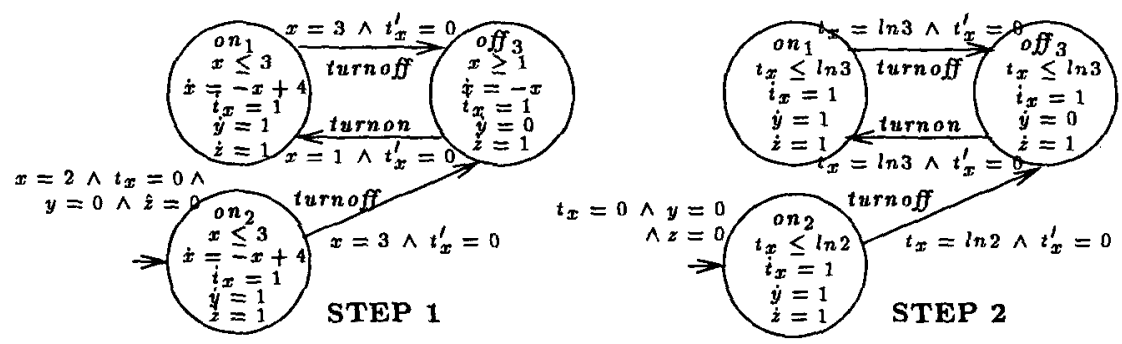

Fig. 3. Clock translation of the thermostat automaton

label label $(e)$; for each definite edge $e=\left(v, v^{\prime}\right)$ with the arrival value $c_{j}$, we introduce all edges of the form $\left(v_{c_{i}}, v_{c_{j}}^{\prime}\right)$ with the action $\operatorname{act}(e) \wedge t_{x}^{\prime}=0$ and the label label $(e)$. For example, the thermostat automaton of Figure 1 has only definite edges. The critical values of $x$ are 1,2 , and 3 , so we split both locations on and off into three locations each. Since the locations $o n_{3}$, off 1 , and off 2 are not reachable by a sequence of automaton edges from the initial location on $n_{2}$, we remove these three locations from the clocktranslated automaton. The result of Step 1 is shown on the left in Figure 3.

Step 2. Updating accepting conditions, invariants, and actions Let the function $x(t)$ be the solution of the initial-value problem " $\dot{x}(t)=\operatorname{dif}(v, x) ; x(0)=c_{i}$ ". We now eliminate the nonlinear variable $x$ from the initial and accepting conditions, invariant, and exit-edge actions of each new location $v_{c_{i}}$.

First, we simply remove all the atomic data predicates that involve the variable $x$ from the initial condition init $\left(v_{c_{i}}\right)$. Second, we translate the accepting condition of location $v_{c_{i}}$. Suppose that $x \leq c$ is an atomic data predicate of the accepting condition final $\left(v_{c_{i}}\right)$ (other atomic data predicates are handled similarly). We find all finite roots $r_{0}, \ldots, r_{k}$ of $x(t)-c$ (count roots with zero derivatives twice). If no such root exists and $c_{i} \leq c$, then $x \leq c$ is always satisfied and we replace $x \leq c$ by true; if no root exists and $c_{i}>c$, then $x \leq c$ is not satisfiable and we replace $x \leq c$ by false. Otherwise, if $c_{i} \leq c$, then $x \leq c$ is satisfied when the value of $t_{x}$ is in any of the intervals $\left[0, r_{0}\right],\left[\overline{r_{1}}, r_{2}\right], \ldots$; if $c_{i}>c$, then $x \leq c$ is satisfied when the value of $t_{x}$ is in any of the intervals $\left[r_{0}, r_{1}\right],\left[r_{2}, r_{3}\right], \ldots$ We therefore replace $x \leq c$ by the disjunction $V_{\left[r_{i}, r_{i+1}\right] \in I} r_{i} \leq t_{x} \leq r_{i+1}$, where $I$ is the set of root intervals during which $x \leq c$ is satisfied. The result can be transformed into disjunctive normal form.

Third, we translate the invariant of location $v_{c_{i}}$. Suppose that $x \leq c$ is a conjunct of the invariant $i n v\left(v_{c_{i}}\right)$ (other conjuncts are handled similarly). If $\vec{c}_{i}>c$, then the arrival value of $x$ does not satisfy the invariant, and thus we remove the location $v_{c_{i}}$. Otherwise, we find the smallest nonnegative finite root $r$ of $x(t)-c$. If such a root $r$ exists, then the automaton control can reside in the location $v_{c_{i}}$ up to $r$ time units, and thus we replace the conjunct $x \leq c$ of the invariant by the conjunct $t_{x} \leq r$. If no such root $r$ exists, then the automaton control can reside in the location $v_{c_{i}}$ forever, and we replace the conjunct $x \leq c$ by true.

Fourth, we translate the actions of all edges that leave the location $v_{c_{i}}$. Suppose that $x \leq c$ is a conjunct of the action $\operatorname{act}(e)$, where $e=\left(v, v^{\prime}\right)$. We find all finite roots $r_{0}, \ldots, \bar{r}_{k}$ of $x(t)-c$ (count roots with zero derivatives twice). If no such root exists and $c_{i} \leq c$, then the edge $e$ is always enabled and we replace the conjunct $x \leq c$ by true; if no root exists and $c_{i}>c$, we remove the edge $e$. Otherwise, if $c_{i} \leq c$, then the edge $e$ is enabled when the value of $t_{x}$ is in any of the intervals $\left[0, r_{0}\right],\left[r_{1}, r_{2}\right], \ldots ;$ if $c_{i}>c$, then $e$ is enabled when the value of $t_{x}$ is in any of the intervals $\left[r_{0}, r_{1}\right],\left[r_{2}, r_{3}\right], \ldots$ For 
each root interval $\left[r_{i}, r_{i+1}\right]$ during which $e$ is enabled, we introduce an edge with the action $\operatorname{act}(e)$ and the label label( $e$ ) except that (1) the conjunct $x \leq c$ is replaced by the conjunct $r_{i} \leq t_{x} \leq r_{i+1}$ and (2) any atomic subformula involving $x^{\prime}$ is removed. In the thermostat example, we have $x_{o n_{1}}(t)=-3 e^{-t}+4, x_{o n_{2}}(t)=-2 e^{-t}+4$, and $x_{o f f}(t)=3 e^{-t}$. Consider the action $x=3$ of the edge from on to off 3 . Since $\ln 2$ is the unique root of $-2 e^{-t}+4-3$, it follows that $x=3$ iff $t_{x}=\ln 2$. Hence we replace the action $x=3$ with the action $t_{x}=\ln 2$. The final result of Step 2 is shown on the right in Figure 3.

Soundness, completeness, and decidability We show that the clock translation is both sound and complete for checking the emptiness of solvable automata. Let $A$ be a solvable hybrid automaton, and let $A^{c}$ be the automaton clock translated from $A$ by translating a nonlinear variable $x$ into a clock $t_{x}$. We show that $A$ and $A^{c}$ are bisimilar.

We first recall the definition of (timed) bisimulation. Let $T_{1}=\left\langle\Sigma_{1}, I_{1}, \mathcal{L}, \rightarrow_{1}\right\rangle$ and $T_{2}=\left\langle\Sigma_{2}, I_{2}, \mathcal{L}, \rightarrow_{2}\right\rangle$ be two labeled transition systems. The binary relation $\approx \subseteq \Sigma_{1} \times \Sigma_{2}$ is a bisimulation between $T_{1}$ and $T_{2}$ if for all states $\sigma_{1} \in \Sigma_{1}$ and $\sigma_{2} \in \Sigma_{2}, \sigma_{1} \approx \sigma_{2}$ implies for every letter $m \in \mathcal{L}$ that (1) if $\sigma_{1} \stackrel{m}{\rightarrow} \sigma_{1}^{\prime}$, then there exists a state $\sigma_{2}^{\prime}$ such that $\sigma_{2} \stackrel{m}{\rightarrow} \sigma_{2}^{\prime}$ and $\sigma_{1}^{\prime} \approx \sigma_{2}^{\prime}$; and (2) if $\sigma_{2} \stackrel{m}{\rightarrow} \sigma_{2}^{\prime}$, then there exists a state $\sigma_{1}^{\prime}$ such that $\sigma_{1} \stackrel{m}{\rightarrow} \sigma_{1}^{\prime}$ and $\sigma_{1}^{\prime} \approx \sigma_{2}^{\prime}$. The two states $\sigma \in \Sigma_{1}$ and $\sigma^{\prime} \in \Sigma_{2}$ are bisimilar if there exists a bisimulation $\approx$ between $T_{1}$ and $T_{2}$ such that $\sigma \approx \sigma^{\prime}$. The labeled transition systems $T_{1}$ and $T_{2}$ are bisimilar, denoted $T_{1} \approx T_{2}$, if for each initial state $\sigma \in I_{1}$, there is a initial state $\sigma^{\prime} \in I_{2}$ such that $\sigma \approx \sigma^{\prime}$, and vice versa. The two hybrid automata $A$ and $B$ are bisimilar if $[A] \approx[B]$.

We define the function $\alpha_{x}: \Sigma_{A^{c}} \rightarrow \Sigma_{A}$ such that $\alpha_{x}\left(v_{c}, \mathbf{s}\right)=\left(v, \mathbf{s}^{\prime}\right)$, where the location $v_{c}$ is split from the location $v$ for the critical value $c$, the states $\mathbf{s}$ and $\mathbf{s}^{\prime}$ agree on all variables except $x$ and $t_{x}$, and $s^{\prime}(x)=x\left(s\left(t_{x}\right)\right)$ if the function $x(t)$ is the solution of the initial-value problem " $\dot{x}(t)=\operatorname{dif}(v, x) ; x(0)=c "$.

Lemma 1. Let $A$ be a solvable hybrid automaton, and let $A^{c}$ be the clock translation of $A$ that results from replacing the nonlinear variable $x$ by the clock $t_{x}$. Then for all states $\sigma, \sigma^{\prime} \in \Sigma_{A^{c}}, \sigma \stackrel{m}{\rightarrow} \sigma^{\prime}$ in $\left[A^{c}\right]$ iff $\alpha_{x}(\sigma) \stackrel{m}{\rightarrow} \alpha_{x}\left(\sigma^{\prime}\right)$ in $[A]$.

It follows that the relation $\left\{\left(\sigma, \alpha_{x}(\sigma)\right) \mid \sigma \in \Sigma_{A^{c}}\right\}$ is a bisimulation between [A] and $\left[A^{c}\right]$, and that $[A] \approx\left[A^{c}\right]$. Since bisimilarity is transitive, if $A^{c}$ results from $A$ by replacing several nonlinear variables with clocks, $A$ and $A^{c}$ are still bisimilar.

Theorem 2. If $A$ is a solvable hybrid automaton and $A^{c}$ is a clock translation of $A$, then $A$ and $A^{c}$ are bisimilar.

It follows that the clock translation is sound and complete for all branching-time properties. In particular, for safety properties, we have the following corollary.

Corollary 3. Let $A$ be a solvable hybrid automaton, and let $A^{c}$ be a clock translation of $A$. If $A$ has an accepting trajectory iff $A^{c}$ has an accepting trajectory.

We conclude that for solving the emptiness problem for the nonlinear automaton $A$, it suffices to solve the emptiness problem for the linear automaton $A^{c}$. The emptiness problem for $A^{c}$, however, can be solved exactly only if the clock translation $A^{c}$ is rational. This gives us the following decidability result, which covers a class of nonlinear hybrid automata, while all previously published decidability results refer to linear hybrid automata [8].

Corollary 4. The emptiness problem is decidable for rationally solvable hybrid automata. 

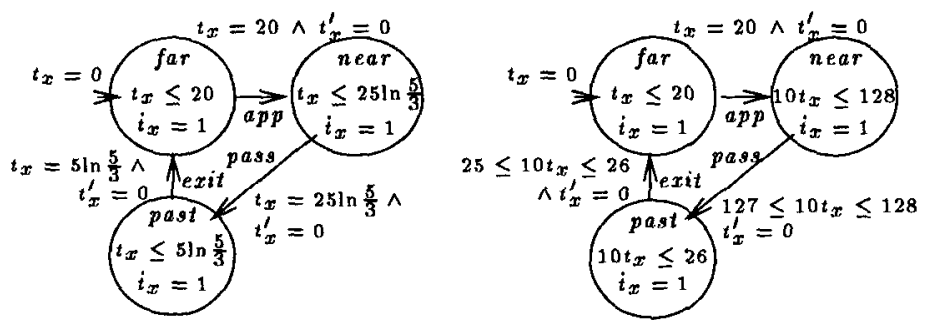

Fig. 4. Clock translation and 0.1-approximate clock translation of the train automaton

$\delta$-approximate clock translation If the clock translation $A^{c}$ is not rational, we approximate $A^{c}$ by a rational automaton, and show soundness for emptiness checking. To preserve soundness when approximating irrational roots numerically, we overapproximate all root intervals. For example, the action $t_{x}=\ln 2$ can be overapproximated by the rational data predicate $693 \leq 1000 t_{x} \leq 694$ with an error bounded by $\delta=1 / 1000$. Formally, a $\delta$-approximation of the data predicate $t_{x} \leq c\left(t_{x} \geq c\right)$, for $\delta \in \mathbb{R}$, is of the form $t_{x} \leq c^{\prime}\left(t_{x} \geq c^{\prime}\right)$ such that $c \leq c^{\prime} \leq c+\delta\left(c-\delta \leq c^{\prime} \leq c\right)$ and $c^{\prime}$ is rational. A $\delta$-approximate clock translation $\left[\bar{A}^{c}\right]_{\delta}$ of $A$ is a rational linear hybrid automaton that is obtained from the clock translation $A^{c}$ by replacing all atomic data predicates in initial and accepting conditions, invariants, and actions by $\delta$-approximations. Note that an action predicate that involves the primed variable $t_{x}^{\prime}$ for a new clock $t_{x}$ are of the form $t_{x}^{\prime}=0$ or $t_{x}^{\prime}=t_{x}$.

We show that $\left[A^{c}\right]_{\delta}$ simulates $A$. To see this, recall the definition of (timed) simulation. Let $T_{1}=\left\langle\Sigma_{1}, I_{1}, \mathcal{L}, \rightarrow_{1}\right\rangle$ and $T_{2}=\left\langle\Sigma_{2}, I_{2}, \mathcal{L}, \rightarrow_{2}\right\rangle$ be two labeled transition systems. The binary relation $\succeq \subseteq \Sigma_{1} \times \Sigma_{2}$ is a simulation of $T_{2}$ by $T_{1}$ if for all states $\sigma_{1} \in \Sigma_{1}$ and $\sigma_{2} \in \Sigma_{2}, \sigma_{1} \succeq \sigma_{2}$ implies for every letter $m \in \mathcal{L}$ that if $\sigma_{2} \stackrel{m}{\rightarrow} \sigma_{2}^{\prime}$, then there exists a state $\sigma_{1}^{\prime}$ such that $\sigma_{1} \stackrel{m}{\rightarrow} \sigma_{1}^{\prime}$ and $\sigma_{1}^{\prime} \succeq \sigma_{2}^{\prime}$. The state $\sigma \in \Sigma_{1}$ simulates the state $\sigma^{\prime} \in \Sigma_{2}$ if there exists a simulation $\succeq$ of $T_{2}$ by $T_{1}$ such that $\sigma \succeq \sigma^{\prime}$. The labeled transition system $T_{1}$ simulates the labeled transition system $T_{2}$, denoted $T_{1} \succeq T_{2}$, if each initial state of $T_{2}$ is simulated by an initial state of $T_{1}$. The hybrid automaton $A$ simulates the hybrid automaton $B$, written $A \succeq B$, if $\llbracket A \rrbracket \succeq[B]$. It is clear that $\left[A^{c}\right]_{\delta}$ simulates $A^{c}$. Moreover, since $A$ and $A^{c}$ are bisimilar, we know that $\left[A^{c}\right]_{\delta}$ simulates $A$.

Proposition 5. Let $A$ be a solvable hybrid automaton. For all $\delta \in \mathbb{R}_{\geq 0}$, if $\left[A^{c}\right]_{\delta}$ is a $\delta$-approximate clock translation of $A$, then $\left[A^{c}\right]_{\delta}$ simulates $A$.

It follows that approximate clock translation is sound for all linear-time properties. Again, for safety properties, we have the following corollary.

Corollary 6. Let $A$ be a solvable hybrid automaton, and let $\left[A^{c}\right]_{\delta}$ be a $\delta$-approximate clock translation of $A$. If $A$ has an accepting trajectory, then so does $\left[A^{c}\right]_{\delta}$.

Example: Railroad gate controller The performance data in this paper was measured on a Sun 670MP workstation. The clock translation $B_{1}^{c}$ of the train automaton $B_{1}$ from Figure 2 is shown on the left in Figure 4, next to a 0.1 -approximate clock translation $\left[B_{1}\right]_{0.1}$ on the right. By taking the product of $\left[B_{1}\right]_{0.1}$ with the gate and controller automata from Figure 2, the HYTECH verifier automatically checks (in 25 seconds of CPU time) that whenever the train is within 100 meters from the gate, then the gate is closed. On the other hand, a 1.0-approximate clock translation of the train automaton is not sufficient for proving this safety property. (After clock translation, the safety property of the thermostat automaton from Figure 1 is checked by HYTECH in 7 seconds of CPU time.) 


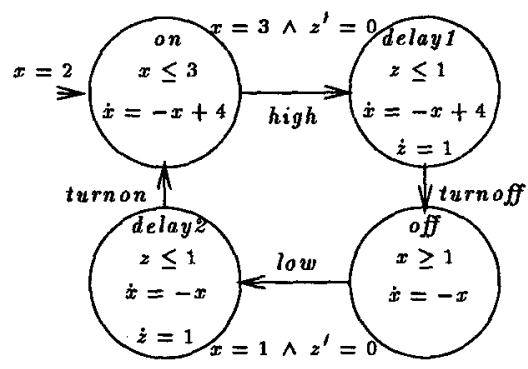

Fig. 5. A temperature controller with delays

Error analysis The approximate clock translation $\left[A^{c}\right]_{\delta}$ may have an accepting trajectory even if $A$ does not. We now show that there is a hybrid automaton that is very close to $A$ and has an accepting trajectory. The following error analysis relaxes all atomic data and action predicates of $A$ to provide an upper bound on the error of $\left[A^{c}\right]_{\delta}$. If the hybrid automaton $A$ models a hybrid system with sensors and actuators, then the $\varepsilon$-relaxed automaton $A^{\varepsilon}$, for $\varepsilon \in \mathbb{R}_{\geq 0}$, models the same system with sensors and actuators that suffer from errors bounded by $\varepsilon$ : (1) all atomic data predicates of the form $x \leq c$ and $x \geq c$ (in initial and accepting conditions, invariants, and actions) are replaced by $x \leq c+\varepsilon$ and $x \geq c-\varepsilon$, respectively; and (2) all atomic action predicates of the form $x^{\prime}=c$ are replaced by $c-\varepsilon \leq x^{\prime} \leq c+\varepsilon$. Notice that if $\varepsilon \rightarrow 0$, then $A^{\varepsilon} \rightarrow A$. We define the metric $d \succeq$ on hybrid automata such that $d \succeq(A, B)$ is the infimum of all nonnegative reals $\varepsilon$ such that $A^{\varepsilon} \succeq B \succeq A$ or $B^{\varepsilon} \succeq A \succeq B$, if such an $\varepsilon$ exists; otherwise, $d_{\succeq}(A, B)=\infty$. The error of the $\delta$-approximate clock translation $\left[A^{c}\right]_{\delta}$ is $d_{\succeq}\left(A,\left[A^{c}\right]_{\delta}\right)$. The following lemma bounds this error.

Lemma 7. Let $A$ be a solvable hybrid automaton, and let $\left[A^{c}\right]_{\delta}$ be a $\delta$-approximate clock translation of $A$. If $\lambda \in \mathbb{R}_{\geq 0}$ bounds the absolute values of the derivatives of all data variables of $A$ in all locations of $A$, then $A^{\delta \cdot \lambda}$ simulates $\left[A^{c}\right]_{\delta} ;$ that is, the error of the $\delta$-approximate clock translation $\left[A^{c}\right]_{\delta}$ is bounded by $\delta \cdot \lambda$.

It follows that the approximate clock translation is asymptotically complete, under the metric $d_{\succeq}$, for checking the emptiness of hybrid automata.

Theorem 8. Let $A$ be a solvable hybrid automaton. For all reals $\varepsilon>0$, there is a real $\delta>0$ such that for all $\delta$-approximate clock translations $\left[A^{c}\right]_{\delta}$ of $A, A^{e} \succeq\left[A^{c}\right]_{\delta} \succeq A$.

Corollary 9. Let $A$ be a solvable hybrid automaton. For all reals $\varepsilon>0$, there is a real $\delta>0$ such that if a $\delta$-approximate clock translation of $A$ has an accepting trajectory, then so does $A^{\varepsilon}$.

\section{Rate Translation}

The rate translation of a hybrid automaton replaces each nonlinear data variable $x$ by a piecewise-linear variable that approximates $x$. The rate translation may be applicable also to unsolvable automata. Consider, for example, the nonlinear hybrid automaton of Figure 5, which models a temperature controller with delays: after the thermometer detects that the temperature is low or high, there may be a delay of up to 1 time unit before the heater is turned on or off. We wish to verify that the plant temperature is always between $\frac{1}{5}$ and $\frac{19}{5}$. The automaton is not solvable, because the edge from delay to off is indefinite for $x$. Hence we cannot apply the clock translation to eliminate 
the nonlinear variable $x$. Instead, we approximate $x$ by a piecewise-linear variable. In location $v$ with the bounded invariant region \inv $(v)$, we bound the derivative of $x$ by its minimum $a$ and its maximum $b$, and then replace the activity $\operatorname{dif}(v, x)$ by the rate interval $[a, b]$. For a better approximation, we split the location $v$ into several locations and limit the size of the rate intervals. Clearly, smaller rate intervals yield a more accurate overapproximation of the automaton trajectories.

Bounded automata Let $A$ be a hybrid automaton. The data variable $x$ of $A$ is nondecreasing (nonincreasing) in location $v$ of $A$ if $i n v(v)$ implies $\operatorname{dif}(x, v) \geq 0$ ( $\operatorname{dif}(x, v) \leq 0$ ). The data variable $x$ of $A$ is bounded with the window $[c, d] \subseteq \mathbb{R}$ if any one of the following three conditions holds:

1. For all states in $R(A)$, the value of $x$ is always within the bounded interval $[c, d]$. In particular, this is the case if for all locations $v$ of $A, \operatorname{inv}(v)$ implies $c \leq x \leq d$.

2. $A$ does not contain constants smaller than $c$ or larger than $d$, and either $x$ is nondecreasing in all locations of $A$, or $x$ is nonincreasing in all locations.

3. $\neg F_{A}$ implies $c \leq x \leq d$.

If the data variable $x$ is bounded with the window $[c, d]$, and its value lies outside $[c, d]$, then the exact value of $x$ is irrelevant for checking the emptiness of $A$. The hybrid automaton $A$ is bounded if all nonlinear variables of $A$ are simple and bounded.

The rate translation algorithm Let $A$ be a bounded hybrid automaton, and let $\delta \in \mathbb{R}_{\geq 0}$ be a nonnegative real. A $\delta$-approximate rate translation $\left[A^{r}\right]_{\delta}$ of $A$ is a linear hybrid automaton that is obtained by the following construction. Consider a data variable $x$ and a location $v$ of $A$. First assume that the activity $\operatorname{dif}(v, x)=f(x)$ is a function of $x$ only. Let $[c, d]$ be the window of $x$. We partition the window $[c, d]$ into $k$ subintervals $I_{1}=\left[c_{0}=c, c_{1}\right], \ldots, I_{k}=\left[c_{k-1}, c_{k}=d\right]$, each of size at most $\delta$. The location $v$ is split into $k+2$ locations $v_{0}, \ldots, v_{k+1}$. Each new location $v_{i}$ has the invariant $\operatorname{inv}(v) \wedge c_{i-1} \leq x \leq c_{i}$, where $c_{-1}=-\infty$ and $c_{k+1}=\infty$. For each $v_{i}$, we compute the minimum $a$ and the maximum $b$ of the function $d i f\left(v_{i}, x\right)$ for $c_{i-1} \leq x \leq c_{i}$. We then approximate the derivative of $x$ in the location $v_{i}$ by the rate interval $\operatorname{dif}\left(\bar{v}_{i}, x\right)=[a, b]$, Finally, we introduce all edges of the form $\left(v_{i}, v_{i+1}\right)$ and $\left(v_{i+1}, v_{i}\right)$ with the action $x=c_{i}$ and the label $v$ (which is the label of the stutter edge $e_{v}$ ); and for each edge $e=\left(v, v^{\prime}\right)$, all edges of the form $\left(v_{i}, v_{j}^{\prime}\right)$ with the action act $(e)$ and the label label $(e)$.

Now consider the general case that $\operatorname{dif}\left(v, x_{i}\right)=f\left(x_{1}, \ldots, x_{n}\right)$. We approximate all nonlinear variables $x_{1}, \ldots, x_{n}$ simultaneously. Suppose that the window for $x_{i}$ is $I_{i}$. We partition $I_{i}$ into $k_{i}$ subintervals $\left[c_{i, 0}, c_{i, 1}\right], \ldots,\left[c_{i, k_{i}-1}, c_{i, k_{i}}\right]$, each of size at most $\delta$. The location $v$ is split into the set $U_{v}=\left\{v\left(a_{1}, \ldots, a_{n}\right) \mid 0 \leq a_{i} \leq k_{i}+1\right\}$ of $\left(k_{1}+2\right) \cdots\left(k_{n}+2\right)$ locations, all with the initial condition init $(v)$ and the accepting condition final $(v)$. The invariant of $v\left(a_{1}, \ldots, a_{n}\right)$ is $\operatorname{inv}(v) \wedge \bigwedge_{i=1, \ldots, n} c_{i, a_{i}-1} \leq x_{i} \leq c_{i, a_{i}}$, where $c_{i,-1}=-\infty$ and $c_{i, k_{i}+1}=\infty$. For each new location, we compute the rate intervals for all $x_{i}$. For each pair $v(\mathbf{a})$ and $v(\mathbf{b})$ of new locations, we introduce all edges of the form $(v(\mathbf{a}), v(\mathbf{b}))$ with the axtion $x^{\prime}=x$ and the label $v$ (many of these edges are inconsistent and can be omitted); and for each edge $e=\left(v, v^{\prime}\right)$, we introduce all edges of the form $\left(v(\mathbf{a}), v^{\prime}(\mathbf{b})\right)$ with the action $\operatorname{act}(e)$ and the label label $(e)$.

Soundness We show that the rate translation is sound for checking the emptiness of bounded automata. We define the onto function $\beta: \Sigma_{\left[A^{r}\right]_{6}} \rightarrow \Sigma_{A}$ such that $\beta\left(v^{\prime}, s\right)=$ $(v, \mathrm{~s})$ if $v_{m}^{\prime} \in U_{v}$. It is clear that for all states $\sigma_{1}, \sigma_{2} \in \Sigma_{\left[A^{r}\right]_{\delta}}$, if $\beta\left(\sigma_{1}\right) \stackrel{m}{\rightarrow} \beta\left(\sigma_{2}\right)$ in [A], then $\sigma_{1} \stackrel{m}{\rightarrow} \sigma_{2}$ in $\left[\left[A^{r}\right]_{\delta}\right]$. Hence the relation $\left\{(\sigma, \beta(\sigma)) \mid \sigma \in \Sigma_{\left[A^{r}\right]_{\delta}}\right\}$ is a simulation of $[A]$ by $\left[\left[A^{r}\right]_{b}\right]$.

Proposition 10. Let $A$ be a bounded hybrid automaton. For all $\delta \in \mathbb{R}_{\geq 0}$, if $\left[A^{r}\right]_{\delta}$ is a $\delta$-approximate rate translation of $A$, then $\left[A^{r}\right]_{\delta}$ simulates $A$. 


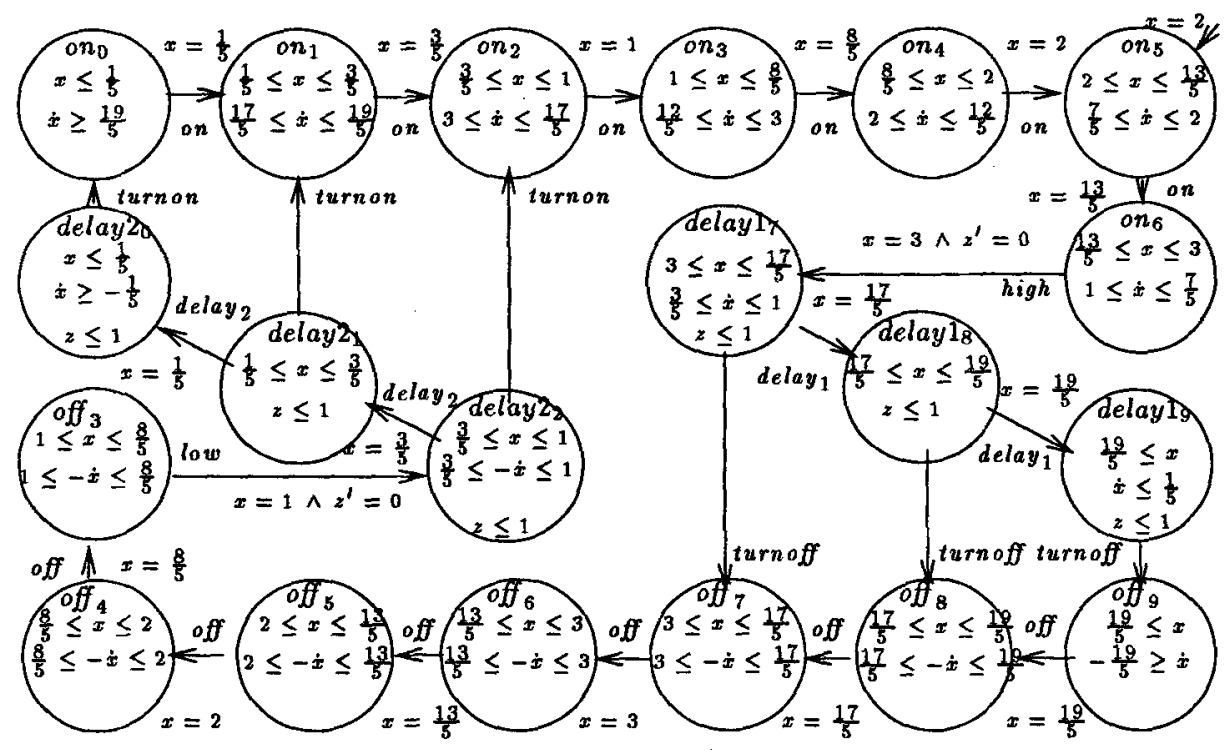

Fig. 6. Rate translation of the temperature controller with delays

It follows that the rate translation is sound for all linear-time properties. In particular, for safety properties, we have the following corollary.

Corollary 11. Let $A$ be a bounded hybrid automaton, and let $\left[A^{r}\right]_{\delta}$ be a rate translation of $A$. If $A$ has an accepting trajectory, then so does $\left[A^{r}\right]_{\delta}$.

Example: Temperature controller with delays Recall the emptiness problem for the automaton $B_{2}$ of Figure 5 with the accepting condition $\bigcup_{v}\left\{\left(v, x \leq \frac{1}{5} \vee x \geq\right.\right.$ $\left.\left.\frac{19}{5}\right)\right\}$. For the rate translation of the automaton $B_{2}$, we partition the window $\left[\frac{1}{5}, \frac{19}{5}\right]$ into the eight intervals $\left[\frac{1}{5}, \frac{3}{5}\right],\left[\frac{3}{5}, 1\right],\left[1, \frac{8}{5}\right],\left[\frac{8}{5}, 2\right],\left[2, \frac{13}{5}\right],\left[\frac{13}{5}, 3\right],\left[3, \frac{17}{5}\right]$, and $\left[\frac{17}{5}, \frac{19}{5}\right]$ of uneven size at most 0.6 (it is a good idea to separate intervals at the point $c$ if $x=c$ is a conjunct of an invariant or action). After removing inconsistent edges and unreachable locations, we obtain the linear hybrid automaton $\left[B_{2}^{r}\right]_{0.6}$ of Figure 6 , which is a 0.6 -approximate rate translation of $B_{2}$. The HYTECH verifier reports that $\left[B_{2}^{r}\right]_{0.6}$ satisfies the safety property that the value of $x$ stays within the interval $\left(\frac{1}{5}, \frac{19}{5}\right)$ (using 45 seconds of CPU time).

Error analysis To analyze the error of the $\delta$-approximate rate translation $\left[A^{r}\right]_{\delta}$, we define the metric $d_{R}$ such that $d_{R}(A, B)$ is the infimum of all nonnegative reals $\varepsilon$ such that $R(A) \subseteq R(B) \subseteq R\left(A^{e}\right)$ or $R(B) \subseteq R(A) \subseteq R\left(B^{e}\right)$, if such an $\varepsilon$ exists; otherwise, $d_{R}(A, B)=\infty$. The error of the $\delta$-approximate rate translation $\left[A^{r}\right]_{\delta}$ is $d_{R}\left(A,\left[A^{r}\right]_{\delta}\right)$, where $R\left(\left[A^{r}\right]_{\delta}\right)$ is interpreted as $\beta\left(R\left(\left[A^{r}\right]_{\delta}\right)\right)$ when compared with $R(A)$. In the full paper, we give an error analysis for the rate translation of monotonic bounded hybrid automata, where in each location each nonlinear variable is nondecreasing or nonincreasing. The rate translation is asymptotically complete, under the metric $d_{R}$, for checking the emptiness of monotonic bounded hybrid automata.

Theorem 12. Let $A$ be a monotonic bounded hybrid automaton. For all reals $\varepsilon>0$, there is a real $\delta>0$ such that for all $\delta$-approximate rate translations $\left[A^{r}\right]_{\delta}$ of $A$, $R(A) \subseteq R\left(\left[A^{r}\right] \delta\right) \subseteq R\left(A^{e}\right)$. 
Corollary 13. Let $A$ be a monotonic bounded hybrid automaton. For all reals $\varepsilon>0$, there is a real $\delta>0$ such that if a $\delta$-approximate rate translation of $A$ has an accepting trajectory, then so does $A^{e}$.

\section{Discussion}

The two translations presented here provide algorithmic methods for verifying safety properties of two classes of nonlinear hybrid systems: solvable systems and bounded systems. Whenever both translations are applicable, the clock translation is generally preferable, because the size of the translated automaton does not depend on the precision of the translation. This work can be extended in two ways, which are discussed in the full version of the paper. First, symbolic model-checking techniques can be used to verify, in addition to safety properties, arbitrary branching-time properties of solvable and bounded systems. Second, the classes of solvable and bounded systems can be generalized by admitting nonlinear variables that are not simple, and the class of solvable systems can be generalized by admitting linear variables whose dynamics is given by rate intervals $[4,11,12]$.

Acknowledgement We thank Howard Wong-Toi for a careful reading.

\section{References}

1. R. Alur, C. Coucoubetis, N. Halbwachs, T.A. Henzinger, P.-H. Ho, X. Nicollin, A. Olivero, J. Sifakis, and S. Yovine. The algorithmic analysis of hybrid systems. Theoretical Computer Science, 138:3-34, 1995.

2. R. Alur, C. Courcoubetis, T.A. Henzinger, and P.-H. Ho. Hybrid automata: an algorithmic approach to the specification and verification of hybrid systems. Hybrid Systems, Lecture Notes in Computer Science 736, pp. 209-229. Springer, 1993.

3. R. Alur and D.L. Dill. A theory of timed automata. Theoretical Computer Science, 126:183-235, 1994.

4. R. Alur, T.A. Henzinger, and P.-H. Ho. Automatic symbolic verification of embedded systems. IEEE Real-time Systems Symposium, pp. 2-11, 1993.

5. J.R. Burch, E.M. Clarke, K.L. McMillan, D.L. Dill, and L.J. Hwang. Symbolic model checking: $10^{20}$ states and beyond. Information and Computation, 98(2):142$170,1992$.

6. P. Cousot and R. Cousot. Abstract interpretation: a unified lattice model for the static analysis of programs by construction or approximation of fixpoints. $A C M$ Symposium on Principles of Programming Languages, 1977.

7. R.L. Grossman, A. Nerode, A.P. Ravn, and H. Rischel, editors. Hybrid Systems. Lecture Notes in Computer Science 736. Springer, 1993.

8. T.A. Henzinger, P. Kopke, A. Puri, and P. Varaiya. What's decidable about hybrid automata? ACM Symposium on Theory of Computing, 1995.

9. T.A. Henzinger, X. Nicollin, J. Sifakis, and S. Yovine. Symbolic model checking for real-time systems. Information and Computation, 111(2):193-244, 1994.

10. X. Nicollin, A. Olivero, J. Sifakis, and S. Yovine. An approach to the description and analysis of hybrid systems. Hybrid Systems, Lecture Notes in Computer Science 736, pp. 149-178. Springer, 1993.

11. A. Olivero, J. Sifakis, and S. Yovine. Using abstractions for the verification of linear hybrid systems. CAV 94: Computer-aided Verification, Lecture Notes in Computer Science 818, pp, 81-94. Springer, 1994.

12. A. Puri and P. Varaiya. Decidability of hybrid systems with rectangular differential inclusions. CAV 94: Computer-aided Verification, Lecture Notes in Computer Science 818, pp. 95-104. Springer, 1994. 